\title{
Statistical Tests of Noise and Harmony in Dark Matter Modulation Signals
}

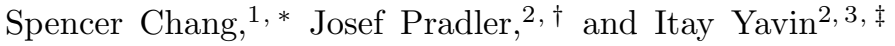 \\ ${ }^{1}$ Department of Physics, University of Oregon, Eugene, OR 97403 \\ ${ }^{2}$ Perimeter Institute for Theoretical Physics 31 Caroline St. N, Waterloo, Ontario, Canada N2L $2 Y 5$. \\ ${ }^{3}$ Department of Physics \&5 Astronomy, McMaster University 1280 Main St. W, Hamilton, Ontario, Canada, L8S 4L8
}

\begin{abstract}
The aim of the current work is a detailed time-series analysis of the data from Dark Matter direct detection experiments as well as related datasets. We examine recent claims that the cosmic ray muon flux can be responsible for generating the modulation signals seen by DAMA and, more recently, by the CoGeNT collaboration. We find no evidence for such a strong correlation and show that the two phenomena differ in their power spectrum, phase, and possibly in amplitude. In addition, we investigate in more detail, the time dependence of Dark Matter scattering. Since the signal is periodic with period of a year (due to the Earth's motion around the Sun), the presence of higher harmonics can be expected. We show that the higher harmonics generically have similar phase to the annual modulation and the biannual mode in particular could provide another handle in searching for Dark Matter in the laboratory.
\end{abstract}

\section{INTRODUCTION}

Whereas the gravitational nature of Dark Matter (DM) is a crucial ingredient for the success of the standard cosmological model, its non-gravitational character proves elusive and remains essentially unknown to date. The possible connection with the electroweak scale and the associated expectation of an interaction strength not much smaller than $G_{F}$ and a DM mass not much lighter than $\mathcal{O}(10 \mathrm{GeV})$ has motivated the construction of experiments looking for the direct scattering of DM against nuclei in the lab [1]. The experimental efforts in the field are proceeding in full force and at least another decade of progress is expected.

With the main observable being the nuclear recoil spectrum the information content is rather limited. Moreover, as was realized over the past decade, the recoil spectrum resulting from DM collisions is model dependent and may not follow the simple exponential rise towards low energies expected from elastic scattering [26. On the other hand, one of the most robust predictions of the cold DM model is that the relative velocity of the Earth against the DM halo should vary with the Earth revolution around the Sun [7, 8. The expected recoil rate is therefore a general periodic function with a fundamental period of one year with a particular phase. Despite the strong motivations to look for such modulations, it has so far been achieved by only two experiments, DAMA [9, 10] and CoGeNT [11]. The difficulty is of course in maintaining the stability of the apparatus and the rejection of background over a long period of time. Indeed, the two experiments just mentioned have experienced considerably more background events than some of the other extremely clean experiments such as CDMS-II [12, 13] and XENON100 [14]. Moreover, the

\footnotetext{
* chang2@uoregon.edu

$\dagger$ jpradler@perimeterinstitute.ca

$\ddagger$ iyavin@perimeterinstitute.ca
}

search for annual modulations is not without difficulties since many more mundane phenomena are known to exhibit such modulations. Therefore, two of the main goals of this paper are 1) to show that the time series of the reported DAMA and CoGeNT signals can be used to make firm statements about background hypotheses proposed in the literature and 2) to establish further observables based on the time distribution of the energy spectrum.

The only direct detection experiment which claims detection of a firm signal from dark matter is DAMA, situated in the underground LNGS laboratory at Gran Sasso, Italy. The DAMA dataset consists of two main periods, DAMA/NaI (Dec 1995 - July 2002) and DAMA/LIBRA (Sept 2003 - Sept 2009), amounting to a cumulative exposure of 1.17 ton $\times$ years. The residual rate shown by the collaboration exhibits a very clear annual modulation compatible with what is expected from DM models where the Earth's motion around the Sun results in a modulation peaking on approximately June $2^{\text {nd }}$. The collaboration has also released the modulation amplitude as a function of recoil energy, which seems to exhibit a peculiar form possibly more consistent with inelastic scattering [2 6]. It should be kept in mind, however, that this energy spectrum is obtained from the full data set by assuming the periodic functional form. Sadly, there has been no release of the full data set to date. It is particularly unfortunate because, as we will see below, the procedure carried by the collaboration to obtain the power spectrum of the signal and other parameters makes it difficult to compare to both background and signal hypotheses.

Given the seasonal nature of the signal it is entirely conceivable that environmental effects induce backgrounds with an annual modulation pattern just like the one seen by DAMA. As such, it is clear that such contamination may depart significantly from a sinusoidal distribution in time. It is therefore important-whenever enough data on a putative background inducing process is available - to assess its viability based on a full timeseries analysis. For example, in this work we employ Pearson's coefficient of correlation as a test statistic when 


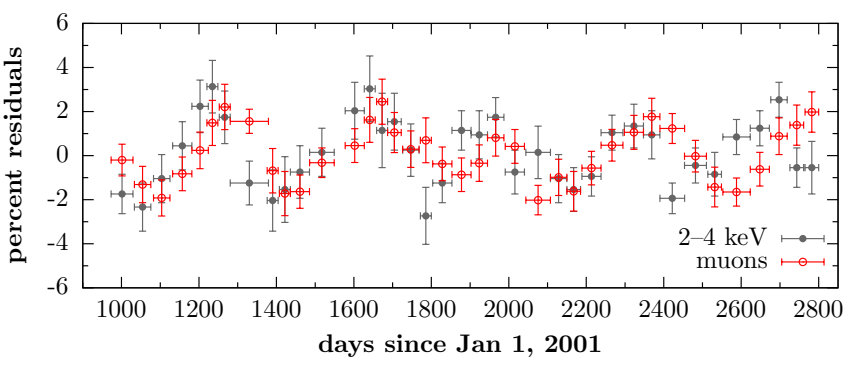

FIG. 1. Percent annual residuals of the LVD measured muon flux when binned in accordance with the DAMA/LIBRA runs $1-5$. The latter residuals are shown for the $2-4 \mathrm{keV}$ bins assuming a baseline count rate of $\bar{s}=1.15 \mathrm{cpd} / \mathrm{kg} / \mathrm{keV}$.

quantifying the compatibility of two data sets. This allows for a quantitative comparison between two data sets which is independent of any assumption about the functional form of the time variations.

Fluxes of neutrons probably constitute the most dangerous source of background as they lead to nuclear recoils which can mimic DM-nucleus interactions [15]. Indeed, it is known from ICARUS measurements at LNGS [16] that the ambient neutron flux generated in the surrounding rock shows some variation on the timescale of a year. However, with a total of five data points we find that it is not possible to make any statistically significant statement regarding the temporal compatibility of this candidate background with the DAMA signal. We therefore choose not to elaborate on this issue any further.

In contrast to rock-generated neutrons, a wealth of data is available on another guaranteed source of neutrons: cosmic ray muons which can penetrate deep underground and induce spallation reactions in the detector and nearby. It is also possible for these muons to deposit their energy directly into the detector crystals [17. Measurements of the muon flux underground have a long history and its seasonal variation is firmly established. There are published measurements at LNGS from MACRO [18, LVD [19] and most recently from Borexino [20]. For DAMA, the relevant measurement is the one from LVD since it was taken at the same underground lab and it overlaps in time with the DAMA/LIBRA runs 1-5. Figure 1 shows the percent residuals of the muon flux when binned in concordance with DAMA with the annual mean count rate subtracted. Also shown are the residuals in the $2-4 \mathrm{keV}$ bin of the DAMA/LIBRA data assuming a baseline rate of $\bar{s}=1.15 \mathrm{cpd} / \mathrm{kg} / \mathrm{keV}$. The seeming similarity in time and amplitude is tantalizing and it has lead various authors to put forward the hypothesis that both signals are in fact measurements of the very same cosmic ray phenomenon [15, 17, 21]. It will be one of the central points of the paper to critically examine these claims, finding that they have difficulty explaining the observed modulations.

The issue of annual modulation in direct DM detection has recently received further impetus from the CoGeNT experiment, located in the Soudan Mine, MN, USA. The collaboration has published its analysis on a 458 day run with a 440 gram Ge detector [11. After removal of cosmogenic background contamination, the data exhibits a seasonal variation peaking around the middle of April. As we shall see below the modulation is manifest only in the higher energy part of the recoil spectrum where the energy spectrum is rather flat with none of the usual features expected from the direct detection signal of DM, elastic or otherwise. The unexplained exponential rise in the recoil spectrum at lower energies, which has stirred quite a commotion in the recent past, shows no evidence of annual modulations. Making use of the time-stamped data provided by the collaboration we address the modulation part of the signal (see also ref. [22, 23] for prior analyses) and investigate the potential role of cosmic ray muons for the CoGeNT detector.

In light of the significant advances in sensitivity from direct detection experiments achieved in the past decade and future improvements expected in the next one, we also address the question of whether the time-series of a signal may encode additional evidence that it is DM which is scattering on a target. In particular we point out that higher harmonics may be present in an annually modulated signal. We show how this signature manifests itself in the scattering rate and explore to some extent its sensitivity on the assumed halo parameters.

The paper is organized as follows: We start with section [II by establishing statistical evidence for periodic variation in the data sets under consideration. Restricting ourselves to a sinusoidal form of the signals, in section III we allow both phase and period to vary and explore the inferred values of these two parameters from the different datasets. In section IV] we drop the assumption of a sinusoidal form and instead directly compare the time series of the various data sets by performing a correlation analysis. Section $\mathrm{V}$ discusses the effect of higher harmonic modulations as a new diagnostic tool when searching for dark matter in the lab. We summarize the main conclusions and findings of the paper in section VI

\section{REJECTING THE NULL HYPOTHESIS}

The existence of a periodic signal in the DAMA and LVD data sets is clear even without any sophisticated statistical analysis. The situation is somewhat more subtle in the case of CoGeNT. Both for the purpose of completeness, and because it reveals interesting differences between the data sets, we begin our analysis by testing the null hypothesis of no signal (i.e. only pure noise) in each of the data sets. The most convenient way of doing such a spectral analysis is by looking at a periodogram of the data.

The classical periodogram of Schuster 24 allows for a calculation of the power spectrum of a signal even in 
the case of uneven sampling of the data. In ref. 25] Scargle showed that a modified version of the classical periodogram, which we call the Lomb-Scargle (LS) power spectrum (and sometimes just power spectrum), results in the same well defined statistical behavior as the Fourier power spectrum used in the case of even sampling. The LS power spectrum allows one to reject noise at a $1-\alpha$ confidence level for a single, preselected frequency by demanding a power level in excess of $z_{\alpha}=\ln \left(\alpha^{-1}\right)$. If one is testing $N$ independent frequencies then the power level required increases to $z_{\alpha}=-\ln \left[1-(1-\alpha)^{1 / N}\right]$ which contains the statistical penalty for inspecting more than one frequency. This means that when testing for the null hypothesis over a range of frequencies we must employ some care in choosing the frequencies to be tested if we want a meaningful statistical interpretation. If the time series is evenly distributed then the standard choice for the frequencies in the discrete Fourier transform results in independent frequencies. Since the data sets we consider are not grossly uneven and approximately cover an integer number of years we chose $\omega_{n}=n \omega_{F}$ with the fundamental frequency $\omega_{F}=2 \pi / T$ where $T$ is roughly the range of years covered by the data set 1 . We checked that this results in low correlation among the test frequencies using the procedure described in App. D of Ref. [25].

\section{A. DAMA and LVD data}

We start by considering the measurements of the muon flux by the LVD experiment [19. The average integral muon intensity underground is reported as $\left\langle I_{\mu}\right\rangle \simeq$ $3 \times 10^{-4} \mathrm{~m}^{-2} \mathrm{sec}^{-1}$ with an annual variation of $\sim 2 \%$ in amplitude as can be seen from Fig. 1. The data spans a total of eight years with more than 2800 data points which we obtain by digitizing Fig. 2 of [19].

The solid line in Fig. 2 shows the power spectrum obtained from the full LVD data set. Aside from the yearly modulation, which is plainly visible from the time series itself, the power spectrum also speaks unequivocally of the existence of temporal variations on time-scales greater than a year. That same figure (dashed line) shows the effect of subtracting the mean intensity from the data on a yearly basis as done by the DAMA collaboration with their own data. The figure makes it clear that such a procedure would mask most of the power at periods much greater than a year. Nevertheless, we note that substantial power remains in modes with periods a little over one year.

\footnotetext{
1 The power spectra in this section all display a peak at a period of exactly one year. We caution the reader that this does not imply the best fit value would be exactly one year, but is simply an artifact of the frequencies we chose to sample when looking to reject the null hypothesis.
}

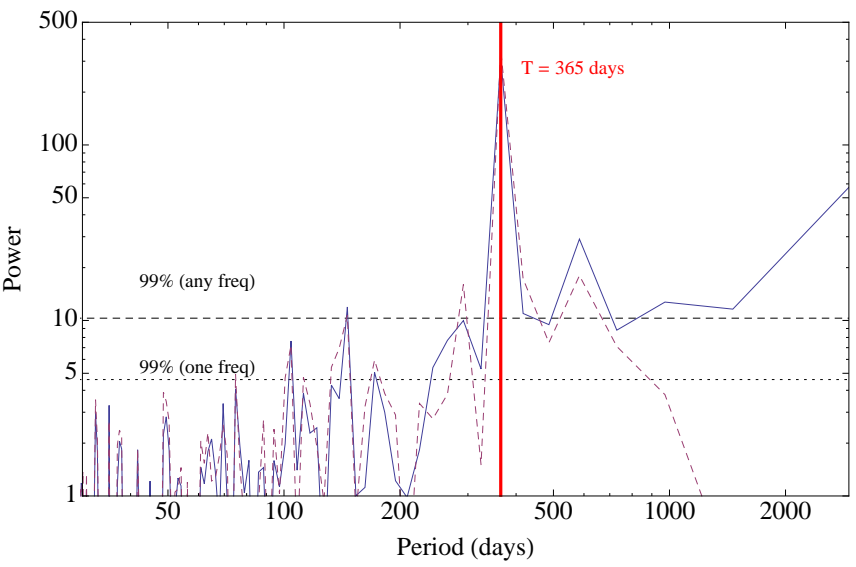

FIG. 2. The Lomb-Scargle power spectrum of the LVD data as a function of the period (solid-blue). Also drawn are the $99 \%$ confidence lines for excluding the noise hypothesis for a single frequency (lower) or any of the frequencies shown (top). Note the substantial power present for modes with a period greater than a year. The dashed-red curve shows the effect of subtracting the yearly mean from the data on a yearly basis, as is done by the DAMA collaboration. The subtraction has little effect on the high frequency modes, but results in a strong damping of the long period modes as can be expected.

Figure 3 shows the power spectrum for the DAMA/LIBRA $2-4 \mathrm{keV}_{\text {ee }}$ data set. In contrast to the LVD spectrum, there is little power at periods greater than a year. This, however, may simply be an artifact of the way the DAMA/LIBRA modulation data is obtained. The DAMA collaboration calculates the residuals by subtracting the mean on a yearly basis for each cycle. As mentioned in the previous paragraph such a procedure tends to dampen power at periods much greater than a year. Nevertheless, the absence of any power even for periods slightly over a year, as seen in the LVD data above, is interesting and serves as the first distinguishing feature between the two data sets. Unfortunately, it is difficult to assign a quantitative significance to this difference without the full, unsubtracted time series. This issue further motivates the release of the unsubtracted data by the DAMA collaboration to allow for a proper comparison of the power spectrum. Finally, we note that no biannual mode ( $\mathrm{T}=1 / 2$ year $)$ is present in the DAMA/LIBRA power spectrum. We will comment on this further in Sec. $\mathrm{V}$ below.

\section{B. CoGeNT data}

The CoGeNT collaboration has released the timestamped data of their 442 live-day run [11 for public use. This makes the computation of the LS diagram in principle straight-forward. However, the data also suffers from background activity of electron capture decays of cosmogenically activated long-lived isotopes. A mea- 


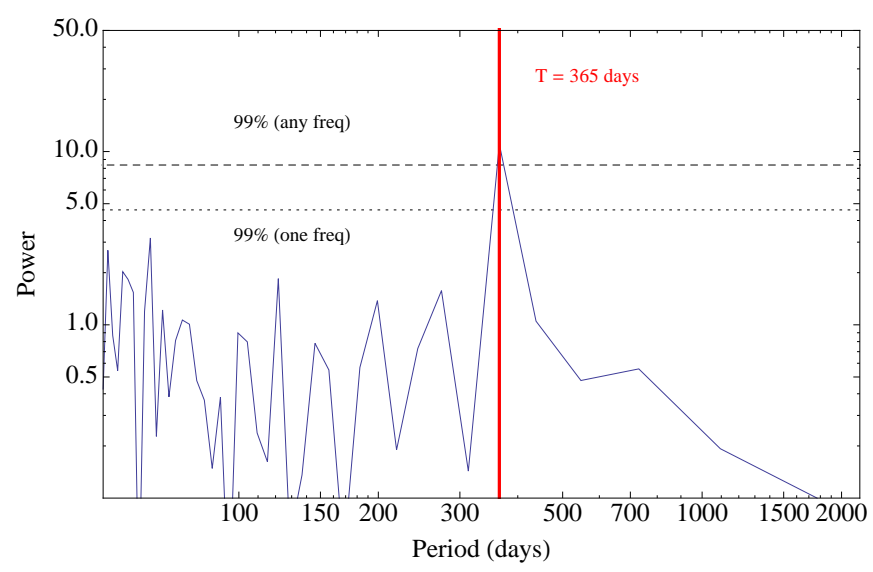

FIG. 3. The Lomb-Scargle power spectrum of the DAMALIBRA data in the $2-4 \mathrm{keV}_{\text {ee }}$ energy region as a function of the period (solid-blue).

surement of higher energetic K-shell captures together with reported ratios of $\mathrm{L}$ - to $\mathrm{K}$-shell decays allows one to correct for the L-shell activity in the DM acceptance region.

Based on the expected cosmogenic activity in the CoGeNT dataset it seems reasonable to divide the low energy data into three regions: $0.5-0.9 \mathrm{keV}_{\mathrm{ee}}, 0.9-$ $1.6 \mathrm{keV}_{\mathrm{ee}}$, and $1.6-3.0 \mathrm{keV}_{\mathrm{ee}}$. Considerable cosmogenic activity is observed in the middle region. In contrast only a small amount of cosmogenic activity is expected in the low region $\left(0.5-0.9 \mathrm{keV}_{\mathrm{ee}}\right)$ and very little if any is expected in the high region $\left(1.6-3.0 \mathrm{keV}_{\mathrm{ee}}\right)$. Since the participating isotopes are rather long-lived $\left(t_{1 / 2} \gtrsim 200\right.$ days $)$ they are expected to result in substantial power at long periods in the corresponding Fourier power spectrum. We have verified that this is indeed the case. However, since we are interested in more localized phenomena in frequency space, such as annual modulations, we need to subtract the cosmogenic activity from the data. We have done this on a daily basis based on the reported activity levels 26. We have verified that the results remain qualitatively the same even when adopting a different subtraction procedure, where we fit for the exponentially decaying component.

Figure 4 shows the power spectrum for the subtracted data in the three energy regions. The power spectrum was calculated in 30 equally spaced frequencies with a fundamental frequency of 2 years. The null hypothesis of noise can only be confidently rejected for the $1.6-$ $3.0 \mathrm{keV}_{\text {ee }}$ region, where considerable power is present at a period of about one year. However, the same cannot be said about the lower energy regions where no significant power is observed. Thus, one can claim the detection of annual modulations at about $99 \%$ confidence level only in the $1.6-3.0 \mathrm{keV}_{\text {ee }}$ region.

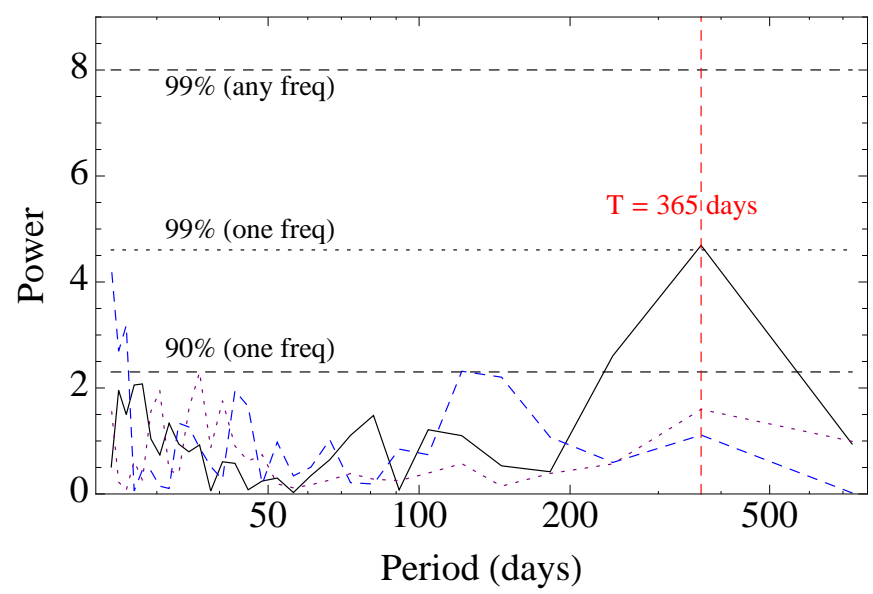

FIG. 4. The Lomb-Scargle power spectrum of the subtracted CoGeNT data as a function of the period for the three energy regions: $1.6-3.0 \mathrm{keV}_{\text {ee }}$ (solid-black), $0.9-1.6 \mathrm{keV}_{\text {ee }}$ (dashedblue), and $0.5-0.9 \mathrm{keV}_{\text {ee }}$ (dotted-purple).

\section{PHASE ANALYSIS}

Aside from the period, the second most important characteristic of the oscillations observed by the DAMA experiment is the phase of the signal. Dark matter collision rate with the detector is expected to peak on June $2^{\text {nd }}$, corresponding to $t_{0}=152$ days after January $1^{\text {st }}$. In this section we investigate the phase associated with the oscillations seen in the DAMA and CoGeNT data and compare them to the phase of the muon intensity modulations. This comparison was already attempted by the DAMA collaboration itself, however, it was criticized by refs. [17, 21] on two accounts. First, in their fit to the data the DAMA collaboration fix the period and allow only the phase to float. Second, the underlying signal may not be truly sinusoidal which may invalidate the statistical inference of a fit to a sine function. We address the first issue in this section and investigate the second problem in the next.

\section{A. Frequentist Approach}

We start with a simple frequentist approach and investigate the effect of departing from strict annual periodicity (i.e $T$ is not necessarily 365 days) on the DAMA phase $t_{0}$. Under the premise of a sinusoidal signal, $A \times \cos \left[\omega\left(t-t_{0}\right)\right]$ with $\omega=2 \pi / T$, we fit amplitude $A$ to the DAMA/LIBRA residuals by minimizing the usual $\chi^{2}$ function while scanning over period $T$ and phase $t_{0}$. A confidence region in $t_{0}$ and $T$ can be constructed from the profile likelihood method [27] which effectively removes the dependence on the nuisance parameter $A$. This way, we first obtain the global minimum $\chi_{\min }^{2}$ from which the 


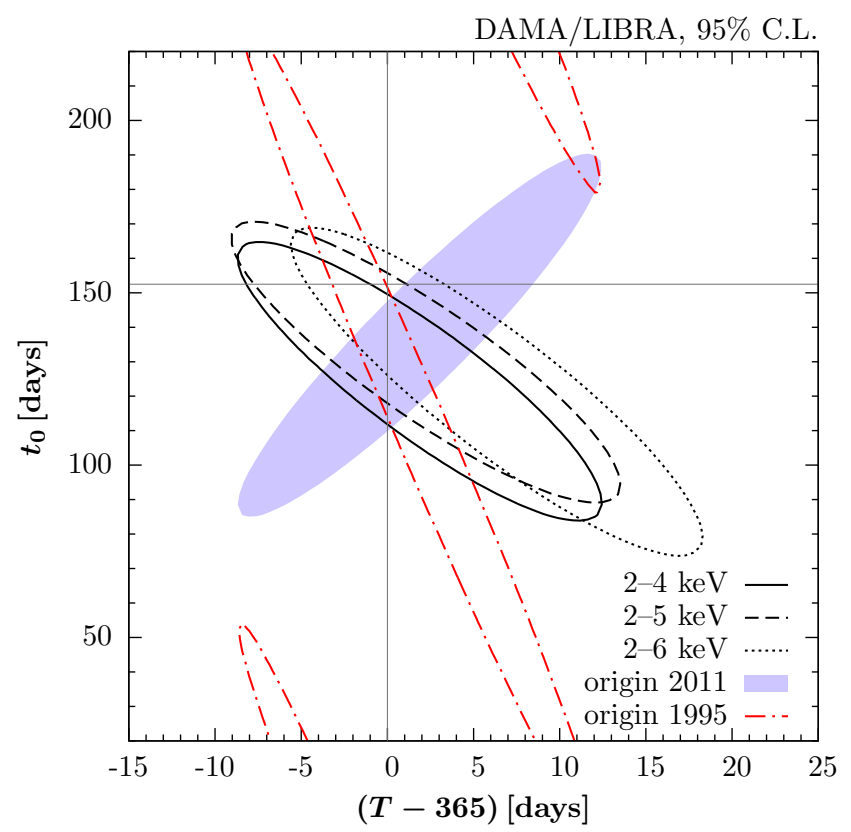

FIG. 5. Confidence regions in period $T$ and phase $t_{0}$ obtained from a $\chi^{2}$ fit to the DAMA/LIBRA residuals. The various lines as labeled correspond to the three different energy binnings provided by the DAMA collaboration with the time origin set to Jan 1, 2003. The shaded region illustrates the effect of shifting the time origin to Jan 1, 2011 for the $2-4 \mathrm{keV}$ residuals and the light dot-dashed ellipse shows the time origin shift to Jan 1, 1995.

confidence region is obtained by requiring

$$
\chi^{2}\left(\hat{A}, t_{0}, T\right) \leq \chi_{\min }^{2}+\Delta \chi^{2},
$$

where $\hat{A}$ is the maximum likelihood estimate for $A$ at each point $\left(t_{0}, T\right)$. For a coverage probability of $95 \%$ one chooses $\Delta \chi^{2}=5.99$. Figure 5 shows the result of fitting the DAMA/LIBRA residuals in the $\left(T, t_{0}\right)$-plane. The three ellipses give the $95 \%$ C.L. regions for the various energy binnings as provided by the DAMA collaboration. As can be seen the data - at the required confidence - are not necessarily consistent with the dark matter interpretation $\left(t_{0}=152\right.$ days and $T=1 \mathrm{yr}$ as indicated by the thin gray lines). As we argue in the next paragraph, the interpretation of such fits have to be handled with some care.

When allowing the period to float it is important to realize that statements about the inferred phase become dependent on the starting date. First, there is the obvious effect that the phase is measured in days with respect to Jan $1^{\text {st }}$, but if the period is not one full year, then which year is used as the origin affects the phase. Comparison of the phase in days between experiments with different origins is meaningless until the origins are made to coincide. This effect is simple to correct for and requires that we use the same time origin for the different data sets. In Fig. 5 the chosen time-origin for the three ellipses showing the various energy bins is Jan 1, 2003.
There is another, more subtle issue that affects the determination of the phase when the period is allowed to float. As can be seen from the ellipses in Fig. 5 the DAMA data exhibits an anti-correlation between the period and the phase. Fits with periods longer than a year strongly favour phases smaller than $t_{0} \sim 150$ days and vice-versa. Note, however, that the sign of the correlation itself depends on the chosen origin. When shifting the latter forward such that the DAMA/LIBRA data lies in the past, $t_{0}$ and $T$ become positively correlated instead. This is illustrated in Fig. 5 by the filled ellipse obtained from the $2-4 \mathrm{keV}$ energy bins for which we have chosen Jan 1, 2011 as the origin. It is important to note that the distribution in $t_{0}$, given a certain period, depends on the chosen time origin. Ideally, we would like to make coordinate-independent statements and care must be taken when interpreting the results. This issue further motivates the correlation study provided in the next section.

Given the above, before proceeding and comparing the LVD and DAMA data sets we must decide on a common time origin. The starting date used by the DAMA collaboration (Jan $\left.1^{\text {st }}, 1995\right)$ is six years apart from the LVD data (Jan $\left.1^{\text {st }}, 2001\right)$. In our analysis we will concentrate on the DAMA/LIBRA data, which started on September $9^{\text {th }}, 2003$, and so a sensible choice is January $1^{\text {st }}, 2003$ as the origin since it has sufficient overlap with both data sets. To quantify the level of agreement of the muoninduced background hypothesis with DAMA we switch now to a Bayesian approach. This allows for a convenient generalization of the Lomb-Scargle periodogram into the two dimensional phase-frequency space as discussed in Appendix A. Such an approach is particularly convenient when one wishes to incorporate further assumptions on the provided data sets (e.g. choosing priors on the period, phase, and amplitude). We will not incorporate any such assumptions in the current analysis, since we would like to keep it as unbiased as possible. Moreover, we have verified that the conclusions arrived at below remain the same when we employ a frequentist analysis instead.

\section{B. Bayesian Approach}

The Bayesian approach can conveniently generalize the Lomb-Scargle periodogram and allow for the testing of different hypotheses and priors. In this section we restrict ourselves to modelling the data with a simple sinusoidal function $f(t)=A \times \cos \left[\omega\left(t-t_{0}\right)\right]$ but following through the steps presented in Appendix A, a generalization to more complicated test-functions is in principle straightforward.

We are mainly interested in the relationship between phase $t_{0}$ and period $T$. The posterior probability $P\left(\left\{\omega, t_{0}\right\} \| d\right)$, i.e. the probability of observing frequency $\omega=2 \pi / T$, and phase $t_{0}$ given the data $d$ is obtained by integrating the full posterior $P\left(\left\{A, \omega, t_{0}\right\} \| d\right)$ over the 
amplitude $A$ restricted to positive values ${ }^{2}$

$$
\begin{aligned}
P\left(\left\{\omega, t_{0}\right\} \| d\right) & \propto \frac{\sigma^{1-N}}{\sqrt{p}}\left[1+\operatorname{Erf}\left(\frac{h}{\sqrt{2 \sigma^{2} p}}\right)\right] \\
& \times \exp \left(\frac{h^{2}}{2 \sigma^{2} p}\right),
\end{aligned}
$$

where,

$$
\begin{aligned}
& p=\sum_{i} \cos ^{2} \omega\left(t_{i}-t_{0}\right), \\
& h=\sum_{i} d_{i} \cos \omega\left(t_{i}-t_{0}\right) .
\end{aligned}
$$

Before proceeding and using (2) in the full twodimensional period-phase space we can make contact with the procedure which is usually employed, i.e. fixing the period to one year, $T=365$ days. Thereby we impose a delta function prior on the period centered at one year from which we consequently obtain the posterior probability in phase only, $P\left(\left\{t_{0}\right\} \| d\right)$. We find that the DAMA/LIBRA and LVD data do not agree with respective values

$$
\begin{aligned}
t_{0}(\mathrm{DAMA}) & =(131 \pm 13) \text { days } \\
t_{0}(\mathrm{LVD}) & =(187 \pm 2) \text { days }
\end{aligned}
$$

The tight error bars on the LVD data may at first seem surprising. To better motivate this number we consider that given the 8 years of data, one can easily determine by eye a phase shift of about 20 days. Statistics allows a further reduction of about an order of magnitude because of the very large number of points available in the dataset. However, it should be kept in mind that the uncertainty quoted is only statistical and does not take into account the possible systematic issues associated with the obvious presence of temporal variations on larger time scales ${ }^{3}$. In fact, using the error bars of the LVD data, the resulting $\chi^{2}$ of a fit to a sinusoidal function is extremely poor. Hence, the numbers quoted above should really only be understood as the best inference on the underlying parameters of a model consisting of a single frequency.

To see if the discrepancy between DAMA/LIBRA and LVD remains when relaxing the assumption of a strict annual periodicity, we now evaluate (2) for the two data sets. The result is shown in Fig. 6. This figure clearly shows that the inferred modulations seen in the two data

\footnotetext{
${ }^{2}$ It is possible to obtain a more compact expression for the posterior without the error function by integrating over all amplitude values both positive and negative. However, that leads to a $\pm \pi$ ambiguity in the phase. While this degeneracy is easily removable by inspection, we prefer to avoid this complication in what follows.

3 Moreover, there is a systematic error associated with the digitization of the data that amounts to an additional uncertainty of a couple of days.
}

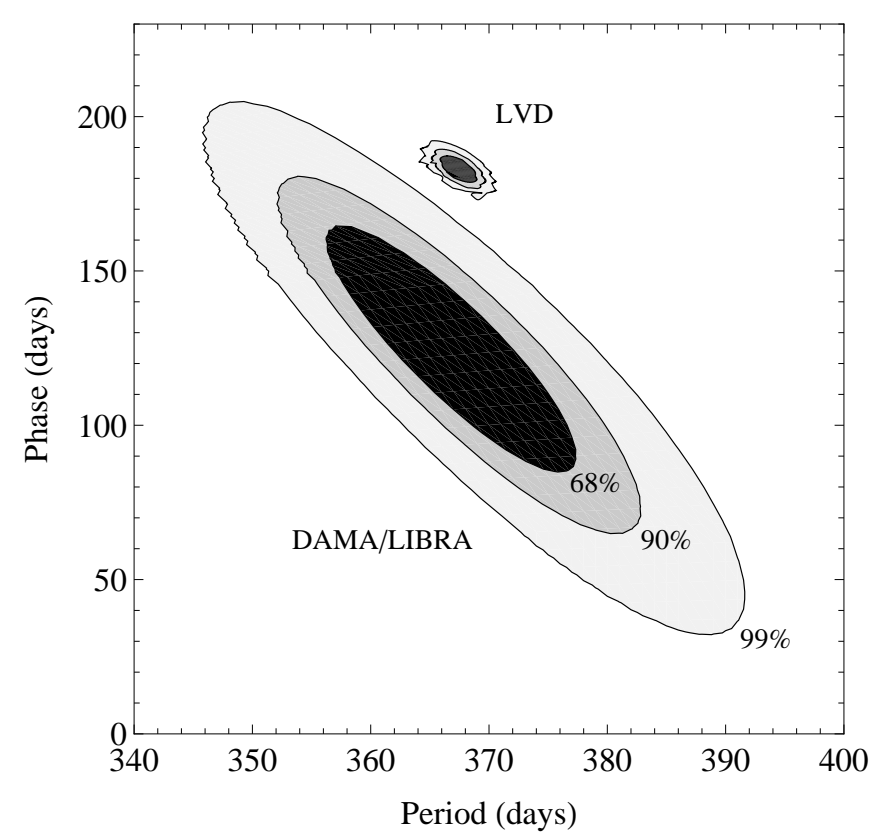

FIG. 6. A comparison of the period-phase posterior for DAMA/LIBRA (bottom) and the LVD phase (top). Shown are the $68 \%, 90 \%$, and $99 \%$ credibility ellipses.

sets - if interpreted as sinusoidal modulations - are incompatible with each other.

A similar analysis can be done for the CoGeNT data 4 as shown in Fig. 7, where we chose to use the entire low energy range $\left(0.5-3.0 \mathrm{keV}_{\mathrm{ee}}\right)$. As discussed in the previous section, evidence for modulation is only present in the restricted range of $1.6-3.0 \mathrm{keV}_{\mathrm{ee}}$. However, isolated power on an annual time scale is present in the entire range and it is therefore not unreasonable to employ the full range when looking to make inferences about the modulation parameters. CoGeNT's credibility ellipses are compared with those obtained from the MINOS data 29]. From there, we arrive at similar conclusions as before, namely, that the muon and direct detection data sets seem incompatible We have verified that when considering the restricted range $1.6-3.0 \mathrm{keV}_{\text {ee }}$ in the $\mathrm{Co}-$ GeNT data we arrive at the same conclusions. We note in passing that the orientation of the MINOS ellipseswhich are obtained with a time origin of Jan $1^{\text {st }}, 2010$ - is similar to the one found in Fig. 5 for DAMA once one shifts the time origin to the future of the data set.

The credibility contours in Fig. 7 indicate a range of viable parameters which is almost twice as large as what has been quoted in the CoGeNT release paper 11. For a direct comparison we thus also perform a frequentist fit to a cosine function (plus a constant.) For example, when using the Minuit package 30] for the $\chi^{2}$-minimization,

\footnotetext{
${ }^{4}$ A Bayesian approach to the CoGeNT data has very recently also been taken by 28 .
} 


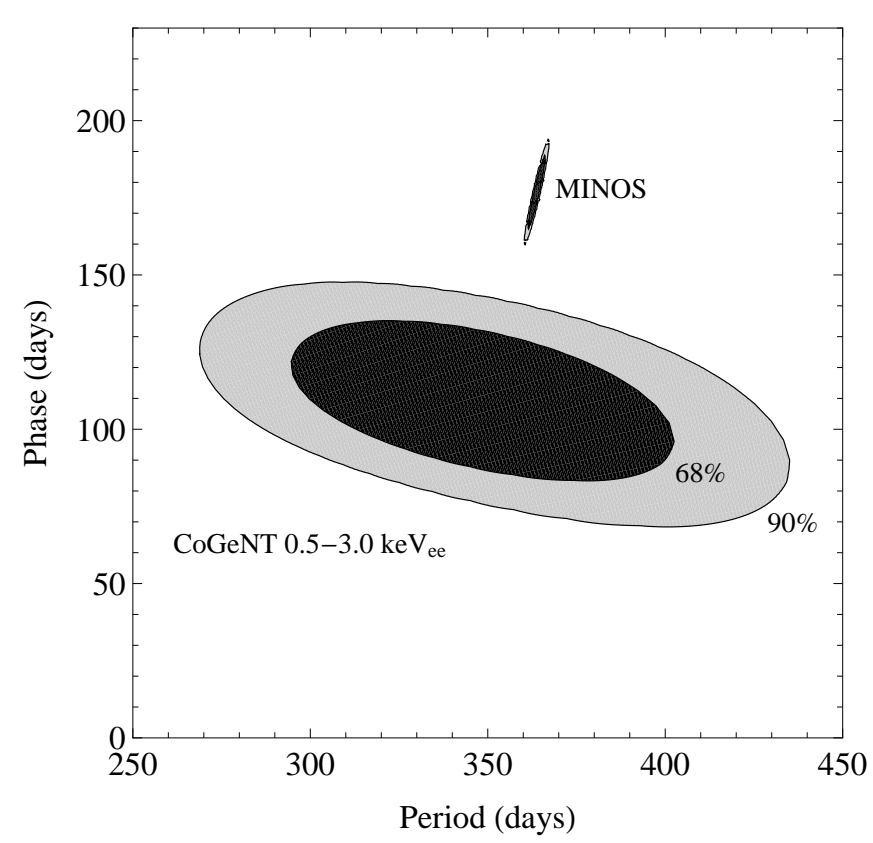

FIG. 7. The period-phase posterior for the full low energy CoGeNT data $\left(0.5-3.0 \mathrm{keV}_{\mathrm{ee}}\right)$ [1] and MINOS data 29]. Shown are the $68 \%$, and $90 \%$ credibility ellipses. The time origin in both cases was chosen to be January $1^{\text {st }}, 2010$.

we obtain $T=350 \pm 26$ days, $t_{0}=110 \pm 13$ days, and $A=17 \% \pm 4 \%$ for a time binning resulting in 21 bins. This is in good agreement with [11. However, it should be stressed that the quoted errors are obtained from a default value of $\Delta \chi^{2}=1$ which does not reflect the increased freedom of fitting more than one parameter. Furthermore $\chi^{2}$ does not grow very large with respect to its rather small minimum value, $\chi_{\min }^{2} /$ d.o.f. $=6.8 / 17$ and even a fit to a constant rate yields a reasonable $\chi_{\min }^{2} /$ d.o.f. $=26.9 / 20$. This can be traced back to large error bars in conjunction with limited data. As a result of this, contours with nominally larger confidence will rapidly open up the parameter space. This, however, does not signal real compatibility of the data sets because of the aforementioned reasons.

\section{CORRELATION ANALYSIS}

A valid concern with regard to the analysis above is the underlying assumption of sinusoidal variation. The power-spectrum of the LVD data, Fig. 2, makes it clear that power exists in modes with periods larger as well as smaller than one year. One may then worry that the phase comparison discussed in the previous section suffers from a systematic misinterpretation. We do not entirely endorse this concern because it is difficult to understand how the very prominent annual modulation in the LVD data, with its very definite phase, can be masked by the much weaker modulations at other frequencies. Nev- ertheless, we now set to investigate this issue in a way that does not rely on assuming any particular functional form for the underlying temporal variations.

Moreover, even if the timing between muons and DAMA (and possibly CoGeNT) are incommensurate at first sight, could it be that the underlying stochastic nature of the background process alleviates the observed tension in the annual phase? This explanation for DAMA was suggested recently in an interesting paper by Blum [21. Assuming a simple, generic model for how a muon-sourced background may be realized, the distribution in phase originating from the Poissonian process is claimed to be compatible with the one observed by DAMA.

In this section we address the above issues by performing a correlation analysis. In particular we follow Ref. 21] and generate mock data for DAMA based on the muon hypothesis. We show that the resulting mock data exhibits a substantially higher degree of correlation with the actual muon data than does the real data from DAMA thus ruling out the hypothesis. We then go on and use a similar correlation analysis to show that the muon hypothesis is also an unlikely cause for the modulations seen by CoGeNT.

\section{A. DAMA}

Since there is considerable overlap between the DAMA/LIBRA and LVD data, it is straightforward to define Pearson's coefficient of correlation. We first bin the LVD data according to the DAMA/LIBRA bins. Then the sample's correlation coefficient is defined as,

$$
r_{X, Y}=\frac{1}{n-1} \frac{\sum_{i=1}^{n}\left(X_{i}-\bar{X}\right)\left(Y_{i}-\bar{Y}\right)}{\sigma_{X} \sigma_{Y}}
$$

where $n$ is the number of overlap bins, $\bar{X}$ and $\bar{Y}$ are the samples' mean, and $\sigma_{X, Y}$ are the samples' standard deviations. The first statistical question we address is whether we can exclude the no-correlation hypothesis. The answer to this question lies in the statistic,

$$
t=\frac{r \sqrt{n-2}}{\sqrt{1-r^{2}}}
$$

which - in the case of the null-hypothesis - is known to follow the Student's distribution with d.o.f $=n-2$. The DAMA/LIBRA and LVD data share 39 temporal bins and so the $90 \%(99 \%)$ two-sided exclusion limit on the statistic is $|t|>1.687(2.715)$. For the correlation between the two data sets we find a value,

$$
r_{\mathrm{LVD}, \mathrm{DAMA}}=0.44 \quad \Rightarrow \quad t_{\mathrm{LVD}, \mathrm{DAMA}}=2.95,
$$

which allows us to exclude the no-correlation hypothesis with confidence level greater than 99\%. This, however, should come as no surprise since both datasets exhibit strong annual modulations with a similar phase. Any such samples will exhibit a correlation at some level, 


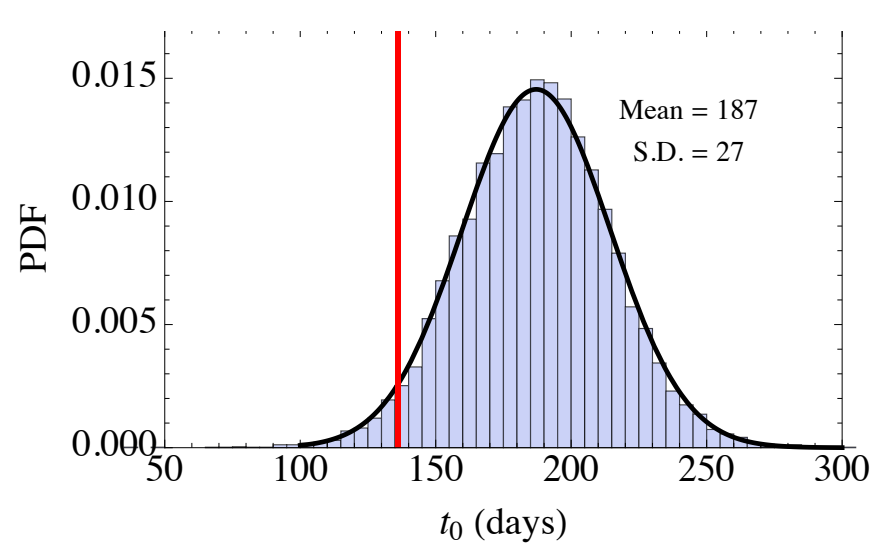

FIG. 8. The population distribution of the phase for the model by Blum, Eq. 10 compared with the best fit to data obtained by the DAMA collaboration, $t_{0}=136$ days, shown as the red vertical line.

but that of course does not imply that they are indeed causally related. The more interesting question we would like to address now is whether we can exclude causation. This question can only be answered in a model-dependent way.

The model we consider was presented by Blum in Ref. [21]. It assumes that the DAMA count rate $s_{i}$ in a time bin of width $\Delta t_{i}$ in an energy range $\Delta E$ for a detector of mass $M$ is related to the muon flux $I_{\mu, i}$ during that time by,

$$
s_{i}=\frac{y N_{\mu, i}}{M \Delta E \epsilon_{i} \Delta t_{i}}
$$

where $N_{\mu, i}$ is Poisson distributed with mean,

$$
\left\langle N_{\mu, i}\right\rangle=A_{\mathrm{eff}} I_{\mu, i} \epsilon_{i} \Delta t_{i}
$$

Here $y$ is the number of signal counts/muon (yield), $A_{\text {eff }}$ is an effective area in which muons are collected, and $\epsilon_{i} \approx 60 \%$ is the duty cycle during time bin $t_{i}$. As was argued in [21], direct hits of muons in the detector would require $y \approx 50$ and would lead to a spread $\sigma_{i} / s_{i}$ in events which is about a factor of five larger than what is actually observed. Since this is in clear conflict with the data, the other possibility that we are going to consider is the secondary effect muons can have due to the spallation reactions on nuclei and the neutron flux they induce. In this case muons can be collected in a larger area, say, $A_{\text {eff }} \approx 10 \mathrm{~m}^{2}$, which requires an order one yield $y \approx 2$ only. Using the latter numbers we generate $10^{4}$ realizations of DAMA data based on this model.

We first attempt to make contact with 21 by plotting in Fig. 8 the distribution of $t_{0}$ obtained from a $\chi^{2}$ fit to a sinusoidal function with floating period, phase, and amplitude. There is little resemblance with the equivalent Fig. 3 presented in 21. The latter shows a very broad distribution with substantial support between $90 \lesssim t_{0} /$ days $\lesssim 250$ and a peak at $t_{0} \approx 100$ days. We find from our Fig. 8 that $t_{0}$ is normally distributed

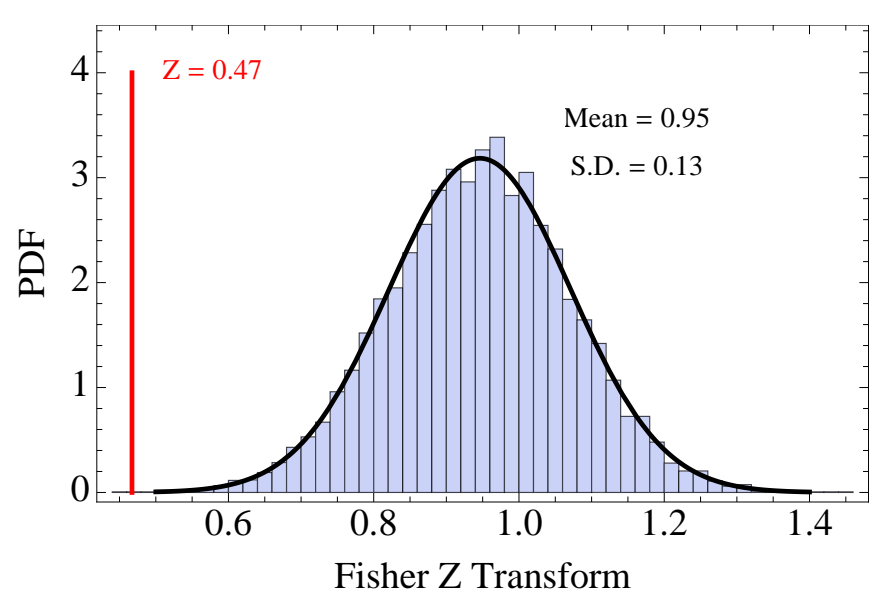

FIG. 9. The population distribution of the Fisher Z-transform for the model by Blum, Eq. 10 as compared to the sample value of $Z=0.47$. Based on the population distribution, the model can be excluded with a confidence exceeding $99 \%$.

with a mean of $\left\langle t_{0}\right\rangle=187$ days and $\sigma=27$ days. We believe that the disagreement with Ref. 21] is due to a different choice of the time-origin. As we discussed in section III once we allow the period to float, the phase $t_{0}$ loses its absolute meaning and the allowed region in $t_{0}$ becomes sensitive to the origin of time. By shifting the origin from Jan $1^{\text {st }} 2003$ to the one DAMA uses when quoting their data, Jan $1^{\text {st }} 1995$, we find that the distribution in $t_{0}$ indeed broadens significantly similar to the one found in [21]. Though it may certainly be better to choose the time origin in 2003, the discrepancy signals a more serious conflict: the distribution in $t_{0}$ does not provide us with a robust test statistic when assessing the compatibility of the mock data with the observed phenomena.

A better way to assess the (in)compatibility between the muon flux and DAMA is to use the generated set of realizations and evaluate the degree of correlation itself. This way we remain completely coordinate independent in our statements and we can attempt to exclude the model by showing that it implies a level of correlation much higher than what is actually implied by the data. This is precisely what we now labour to show. In general, if the model allows an exact calculation of the population correlation coefficient $r_{0}$ then the Fisher $\mathrm{Z}$ transform,

$$
Z=\frac{1}{2} \log \left(\frac{1+r}{1-r}\right)
$$

is approximately normally distributed with mean and standard deviation given by,

$$
\bar{Z}=\frac{1}{2} \log \left(\frac{1+r_{0}}{1-r_{0}}\right) \quad \sigma_{Z}=\frac{1}{\sqrt{n-3}} .
$$

We will not use these results below since the model we consider does not easily allow for an analytic evaluation of the population correlation coefficient $r_{0}$. Instead we 
will utilize the numerical realizations of the model and calculate the distribution explicitly. As it turns out, however, the numerical results nicely agree with the theoretical expectations for the shape of the distribution.

Figure 9 shows the results of the computation of the Fisher Z-transform for each of these realizations. The distribution is compared to the sample's Z-transform $Z=0.47$ obtained from (9). The numerical realizations reveal a much larger expected correlation than what is observed in the data. Therefore the model is excluded with a confidence greater than $99 \%$.

\section{B. CoGeNT}

We now move on to investigate the hypothesis that the reported CoGeNT signal is caused by cosmic muons. In this respect, it is important to note that the apparent modulation fraction of the CoGeNT signal is most prominent in the high-energy bin $1.6-3.0 \mathrm{keV}_{\text {ee }}$ with a value in excess of $10 \%$ [11, 22]. Seasonal muon-flux modulations of that order have indeed been reported by IceCube 31. However, the (somewhat) milder climatic conditions at the Minnesota Soudan Mine location exhibit a variation of at most $4 \%$ 29]. Therefore, unless the scaling of the signal is - for some unknown reason-non-linear in incident muon flux or unless the performance of the detector was not stable throughout the data taking period, it is not possible to generate, say, a 16\% CoGeNT modulation from a lesser modulated sourcing process. This statement is independent of the potential presence of further background. Even though this argument makes a muon explanation of CoGeNT rather unlikely, we can still proceed and look for a quantitative answer based on the temporal structure alone.

The main complication associated with such an analysis is the fact that although measurements of the underground muon flux are available from the MINOS experiment 29], the data has no temporal overlap with CoGeNT. We circumvent this issue by relying on nearby atmospheric temperature data rather than on measurements of the muon flux itself. As is well known, the underground muon flux is tightly correlated with the (stratospheric) temperature. We therefore choose to directly evaluate Eq. (7) between the effective atmospheric temperature parameter $T_{\text {eff }}$ and the background subtracted CoGeNT data. We refer the reader to Appendix $\mathrm{B}$ for further details on this procedure.

A correlation analysis is particularly appropriate in this case since CoGeNT's relatively short data taking period (458 days) naturally limits the significance of any statements about the annual periodicity of the signal as evinced in Fig. 7. However, the correlation analysis we employ requires binning the data, a procedure that is complicated by the need to subtract the cosmogenic background which is responsible for the majority of the event rate in the $0.9-1.6 \mathrm{keV}_{\text {ee }}$ region of the CoGeNT data. One may worry that such subtractions introduce a

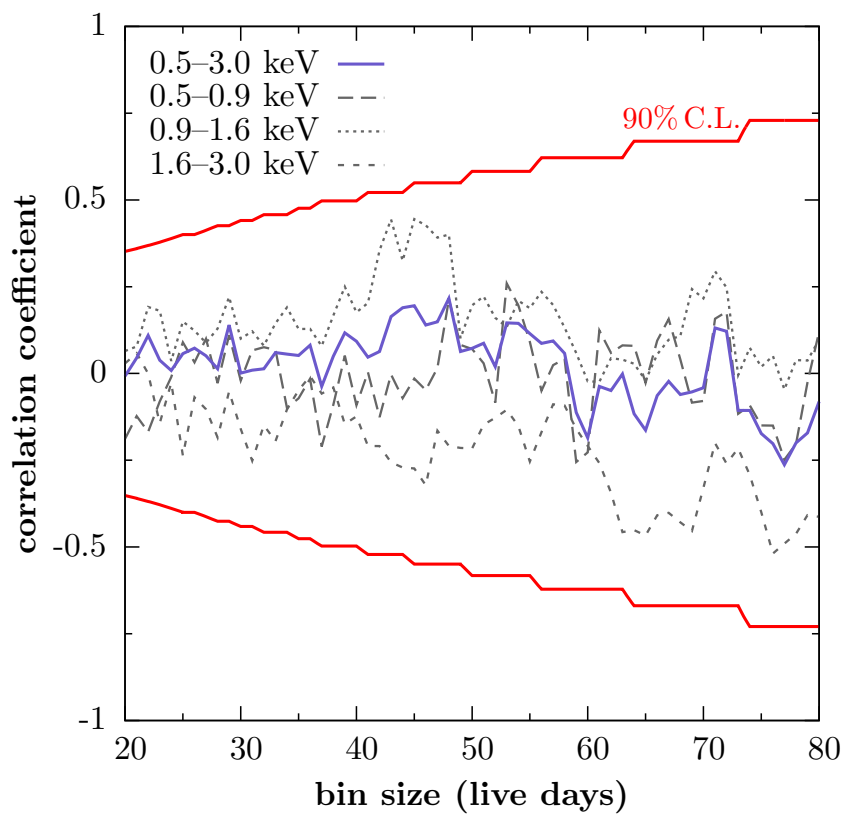

FIG. 10. Coefficent of correlation $r$ between $T_{\text {eff }}$ and CoGeNT data as a function of bin size in live days. The various fluctuating lines correspond to different binnings in energy. None of the chosen energy regions show any significant degree of systematic, positive correlation.

bias depenedent on the choice of binning or energy range. Moreover, the unmodulated component of the subtracted CoGeNT data exhibits a rise towards low energies that is not shared by the modulated part, which if anything seems larger at higher energies. Thus, it is not even clear which part of the energy spectrum could be a result of the muon flux. In light of these complications we consider various time- and energy binning in the hope of thoroughly covering the different possibilities.

Figure 10 shows the correlation coefficient as a function of bin size in live days for various energy ranges. If any (positive) correlation was present, which would corroborate the hypothesis that muons are responsible for the observed signal, one would expect significant degree of correlation independent of the bin size. With the coefficient of correlation fluctuating near zero, the opposite is observed. The step-like red lines delineate the (twosided) $90 \% \mathrm{CL}$ for rejecting the null-hypothesis. The data is therefore perfectly consistent with the null hypothesis of no correlation. For larger bin sizes $r$ begins to fluctuate more strongly. Although it is easier to reach some degree of correlation with fewer bins it correspondingly becomes harder to reach a given level of significance which is indicated by the opening of the red lines. 


\section{BIANNUAL MODULATIONS}

As discussed throughout this work, one of the hallmarks of dark matter direct detection is the annual modulation in the recoil rate, which is the result of the Earth's motion with and against the WIMP wind 7, 8. However, as we show in this section, one also expects higher harmonics (e.g. a period of half a year) to be present at some level and those may prove useful as additional confidence builders if a signal is detected.

\section{A. Harmonic Analysis}

The differential recoil rate, $d R / d E_{R}$ is a function of the Earth's velocity relative to the rest frame of the dark matter halo, which using the notation of Ref. [32] is approximately given by,

$\mathbf{v}_{\text {obs }}=\mathbf{v}_{\odot}+V_{\oplus}\left(\hat{\varepsilon}_{1} \cos \omega\left(t-t_{1}\right)+\hat{\varepsilon}_{2} \sin \omega\left(t-t_{1}\right)\right)$

Here $\mathbf{v}_{\odot}$ is the velocity of the sun relative to the halo, and $\hat{\varepsilon}_{1}\left(\hat{\varepsilon}_{2}\right)$ is the velocity unit vector of the Earth's motion at $t_{1}=$ March $21^{\text {st }}\left(t_{1}+\right.$ year $\left./ 4\right)$. Due to Earth's orbit, the second term proportional to the Earth's relative velocity to the sun, $V_{\oplus} \sim 30 \mathrm{~km} / \mathrm{s}$, modulates with frequency $\omega=\frac{2 \pi}{1 \text { year }}$. If the dark matter velocity distribution is isotropic, then the dark matter speed distribution in the earth's frame, $f(v)$, depends solely upon the magnitude of $\mathbf{v}_{\text {obs }}$. To a good approximation the magnitude of the velocity is given by

$$
\left|\mathbf{v}_{\text {obs }}\right| \approx\left|\mathbf{v}_{\odot}\right|+\frac{1}{2} V_{\oplus} \cos \omega\left(t-t_{0}\right) .
$$

Thus, dark matter scattering rate is a periodic function with a fundamental period of 1 year, but like any periodic function it may contain higher harmonic modulations of any frequency $\omega_{n}=n \omega$. We therefore expect modulations in the scattering rate with periods of $1 / 2,1 / 3, \ldots$ a year corresponding to biannual, triannual and higher frequency modulations. In addition, there are harmonic corrections to $\left|\mathbf{v}_{\text {obs }}\right|$ itself. They arise predominantly from corrections to (14) due to smaller effects such as the ellipticity of the earth's motion around the sun. Once these are taken into account, they result in observable phase shifts between the different harmonics.

We can learn more about the higher harmonics, by expanding in the parameter $\epsilon_{v}=V_{\oplus} / 2 v_{\odot} \approx 0.06$. The time dependence of scattering arises solely due to the velocity dependence through

$$
\begin{aligned}
\frac{d R}{d E_{R}} \propto & \int_{v_{\min }}^{\infty} \frac{f(v)}{v} d v \\
& \approx \sum_{n=0,1, \ldots} \tilde{c}_{n}\left(v_{\min }\right)\left[\epsilon_{v} \cos \omega\left(t-t_{0}\right)\right]^{n} \\
& =\sum_{n=0,1, \ldots} c_{n}\left(v_{\min }\right) \cos \left[n \omega\left(t-t_{0}\right)\right]
\end{aligned}
$$

Here we have used the fact that trigonometric identities allow powers of $\cos \left[\omega\left(t-t_{0}\right)\right]$ to be re-expressed as sums of $\cos \left[n \omega\left(t-t_{0}\right)\right]$. In Eq. (16), $v_{\text {min }}$ is the minimum dark matter speed in the lab which can deposit a recoil energy $E_{R}$

$$
v_{\min }=\frac{1}{\sqrt{2 m_{N} E_{R}}}\left(\frac{m_{N} E_{R}}{\mu_{N \chi}}+\delta\right)
$$

for a nuclear target of mass $m_{N}$ and dark matter-nuclei reduced mass $\mu_{N \chi}=m_{N} m_{\chi} /\left(m_{N}+m_{\chi}\right)$; in (17) we have included the possibility of inelastic scattering with a splitting $\delta$ in the dark matter sector [2, 5. The computation of the harmonic coefficients requires a choice of velocity distribution, and in what follows we consider the distribution proposed in 34,

$$
f_{k}(v) \propto\left[\exp \left(\frac{v_{\mathrm{esc}}^{2}-v^{2}}{k v_{0}^{2}}\right)-1\right]^{k} \Theta\left(v_{\mathrm{esc}}-v\right)
$$

Here, $\Theta(x)$ is the Heaviside step function, and we consider the dispersion $v_{0}=220 \mathrm{~km} / \mathrm{s}$ [35 37] and the escape velocity $v_{\text {esc }}=600 \mathrm{~km} / \mathrm{s} \mathrm{38}$.

In Fig. 11 (top), we illustrate the behavior of the harmonic coefficients, $c_{n}$, for the $k=1.5$ velocity distribution. In order to plot the amplitudes on a logarithmic scale, we have taken the absolute values of $c_{n}$. The troughs in the plots indicate when $c_{n}$ is changing sign. At high enough minimum velocity, $v_{\min }$, the harmonic coefficients are all positive. Interestingly, there are $n$ sign changes for $c_{n}$, which can be understood from the behavior of the velocity distribution $f(v)$.

In the approximation of Eq. (16), the phases of all the higher harmonics are the same as the annual modulation. However, as mentioned above, there are higher order corrections to the magnitude of the observer's velocity, Eq. (15), that allow the phases of the higher harmonics to deviate from the phase of the annual modulation. Indeed, using an accurate parameterization of the earth's velocity 33 , we find appreciable temporal shifts of the biannual and triannual mode with respect to the annual one. This is shown in the middle panel of Fig. 11. The abrupt changes seen in the figure indicate sign changes in the harmonic coefficients $c_{n}$, which can be thought of as phase shifts by $1 \mathrm{yr} / 2 \mathrm{n}$. The ambiguities in the phase shifts are fixed by choosing the values which are closest to the annual one. These phase shifts serve as an additional signature which can be used to discriminate a potentially positive signal from other sources.

In the bottom plot of Fig. 11, we plot the modulation amplitude ratio $c_{n} / c_{1}$ for the biannual and triannual modulation divided by the annual modulation. There are two regions where the higher harmonics are as important as the annual modulation. The first region occurs near $200 \mathrm{~km} / \mathrm{s}$ around the zero in the annual modulation coefficient $c_{1}$. Here, the biannual amplitude $c_{2} / c_{0}$ is at the per mille level which is small but potentially observable with a large amount of exposure. The second region where higher harmonics are important is at high veloci- 
ties, above the escape velocity cutoff. At these high velocities, there are substantially less dark matter particles in the winter than in the summer. Thus, the scattering rate can vanish entirely in winter, with a chopped cosine behavior, which requires more harmonics to fully describe the approach to zero. Although this would be obvious in a experiment with no background, the presence of background can mask these higher harmonics by eye, so it is useful to look for them.

We have also looked at the behavior of these modulation amplitudes for the Standard Halo Model and also for a $k=3.5$ distribution [34. The behavior at low $v_{\min }$ is very similar, since that region depends mostly on the velocity dispersion and not on the behavior near the tail. The high velocity behavior enhances (suppresses) the higher harmonics for sharper (shallower) escape velocity cutoffs as seen in the $k=3.5$ distribution (Standard Halo Model).

A detailed analysis of the detectability of these higher harmonics and their ability to be faked by backgrounds is beyond the scope of this paper. Here, we restrict ourselves to a few remarks. Any background that modulates annually, with a small modulation amplitude will, through the same Taylor expansion argument, also have higher harmonics (although not necessarily all with similar phase). What is peculiar about dark matter is that the higher harmonic amplitudes are enhanced at high $v_{\min }$ due to the escape velocity physics. This could be mimicked by background only if there was some effect during winter which severely suppressed the background appearing as a nuclear scattering event.

On the theory side, models with inelastic dark matter scattering lead to enhanced higher harmonic amplitudes as compared with standard elastic dark matter. A splitting $\delta \sim 100 \mathrm{keV}$ leads to values of $v_{\min }$ which can be near the cutoff (see Eq. 17). In fact, we find that the biannual amplitude can be as large as $30 \%$ of the annual modulation amplitude for the $2-4 \mathrm{keV}_{e e}$ bins of DAMA. Looking at Fig. 3 , we see that this is consistent with the amount of biannual power seen in these bins, since the power ratio between biannual and annual modulation is $\left(c_{2} / c_{1}\right)^{2} \lesssim 0.1$. With a large increase in exposure, by a factor of $O(10-100)$, DAMA would have enough statistics to begin testing this and perhaps even see evidence for a nonzero biannual amplitude as we show quantitatively in the next subsection.

As a final remark, we note that it is possible to predict the energy bin where the annual modulation should be suppressed, due to its change of sign, which would be an interesting bin to look for biannual modulation. For heavy dark matter that scatters elastically, the dependence on the dark matter mass drops out of the reduced mass, so we can use Eq. 17, to show that the energy bin $E_{R}=90 \mathrm{keV}\left(m_{N} / 100 \mathrm{GeV}\right)$ is where the annual modulation should be suppressed. Such high energies might be hard to get substantial dark matter rates though, since the nuclear form factors tend to suppress such high energy recoils. The situation gets better for
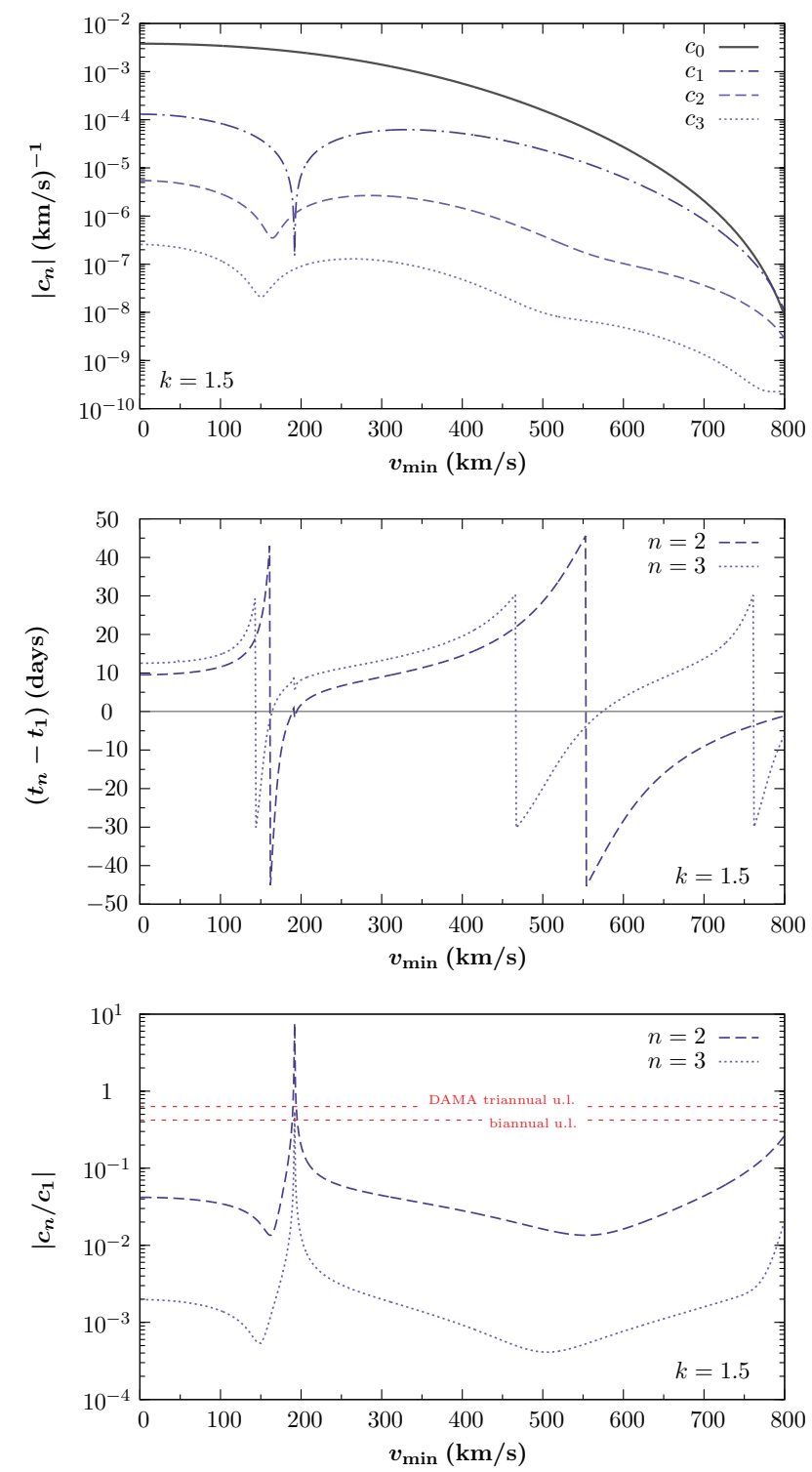

FIG. 11. The velocity dependence of dark matter scattering as a function of $v_{\text {min }}$ for the $k=1.5$ velocity distribution proposed in 34, with dispersion $v_{0}=220 \mathrm{~km} / \mathrm{s}$ and escape velocity $v_{\text {esc }}=600 \mathrm{~km} / \mathrm{s}$. The top plot shows the $\left|c_{n}\right|$ values for $\mathrm{n}=0,1,2,3$ (top to bottom) where $c_{n}$ is in units of $(\mathrm{km} / \mathrm{s})^{-1}$, the middle plot is the phase shift of the higher harmonics relative to the annual modulation, while the bottom plot shows the modulation amplitudes $\left|c_{n} / c_{1}\right|$ for $\mathrm{n}=2,3$ (top to bottom). The horizontal lines in the bottom panel show the $90 \%$ C.L. upper limits on a bi- and triannual signal in the DAMA data.

dark matter much lighter than the nucleus, as the energy bin is $E_{R}=0.9 \mathrm{keV}\left(m_{\chi} / 10 \mathrm{GeV}\right)^{2}\left(m_{N} / 100 \mathrm{GeV}\right)^{-1}$. This requires knowledge of the dark matter mass to predict, but experimentally it might be verified that the sign of the annual modulation amplitude changes sign in a certain bin, thus it would be an interesting followup to see if 
there is a biannual mode in that bin. In fact, the DAMA annual modulation amplitude is smaller in the first bin $2-2.5 \mathrm{keV}_{\text {ee }}$ bin, so it would be interesting to see if there is any biannual modulation there.

\section{B. Testing the Signal Hypothesis}

In the presence of a signal, the probability distribution function of the power $P$ in a particular frequency of the Lomb-Scargle power spectrum changes to [25, 39],

$$
p\left(P ; P_{s}\right)=I_{0}\left(2 \sqrt{P P_{s}}\right) \exp \left(-P-P_{s}\right) .
$$

This formula assumes a normalized signal with power $P_{s}$ that is measured in units of the variance of the noise. Given an observation of power $P_{\text {obs }}$ we can reject a signal at a level of $1-\alpha$ by requiring that,

$$
\operatorname{Pr}\left(P>P_{\mathrm{obs}}\right)=1-\int_{0}^{P_{\mathrm{obs}}} p\left(P ; P_{s}\right) d P=1-\alpha
$$

So, for example, from Figure 3, the DAMA/LIBRA data in the $2-4 \mathrm{keV}_{\text {ee }}$ energy range has a power of $P_{\mathrm{obs}}=0.57$ (1.8) in the biannual (triannual) mode. This implies a frequentist $90 \% \mathrm{CL}$ upper limit of $P_{s}^{\text {biannual }}<1.9(4.4)$ on the power of the biannual (triannual) mode of a putative signal. Since DAMA claims detection of an annually modulating signal we can find the ratio of the higher harmonic power to the annual power. The power observed in the annual mode of the same energy bin is 11.0. Therefore the fractional power in the biannual or triannual mode must be lower than,

$$
\begin{aligned}
& P_{s}^{\text {biannual }}<0.17 P_{s}^{\text {annual }}, \\
& P_{s}^{\text {triannual }}<0.4 P_{s}^{\text {annual }} \text { at } 90 \% \mathrm{CL} .
\end{aligned}
$$

Since the power is directly related to the square of the harmonic coefficient, this limit implies a corresponding limit on the harmonic coefficients, $c_{2} / c_{1}<0.42$ and $c_{3} / c_{1}<0.63$. These limits are shown in the bottom panel of Fig. 11. A similar analysis for the muon flux gives a $90 \%$ CL upper limit on the biannual mode of $P_{s}^{\text {biannual }}<6.3$, see Figure 2. Since the power observed in the annual cycle is $P_{s}^{\text {annual }}=312$ this implies a limit on the harmonic coefficients of $c_{2} / c_{1}<0.17$.

\section{CONCLUSIONS}

This paper was devoted to a detailed time-series analysis of Dark Matter direct detection data as well as related datasets. The main findings are as follows: there is no evidence to support the idea that atmospheric muons are responsible for the signal that the DAMA collaboration observes; the annual modulations observed in the CoGeNT data are statistically significant only in the higher part of the low energy spectrum $\left(1.6-3.0 \mathrm{keV}_{\mathrm{ee}}\right)$; no correlation of the latter with the expected muon flux is observed; biannual modulations may serve as an additional handle on any putative dark matter signal.

With regards to the muon hypothesis discussed in refs. [15, 17, 21] whereby atmospheric muons reaching the DAMA detector are responsible for the signal observed, our analysis indicates three significant difficulties with this idea:

1. The power spectrum of the LVD data on the muon flux indicates considerably more power at periods longer than one year compared with the DAMA/LIBRA data. This remains true even after we follow the undesirable procedure of the DAMA collaboration whereby the residuals are computed by subtracting the mean of the data on a yearly basis. This procedure is undesirable since it masks power at long periods. We therefore urge the DAMA collaboration to release the unsubtracted data to allow for a full comparison of the power spectrum. Similar conclusions have been reached in 21].

2. When fitting the two datasets to a sinusoidal function with variable amplitude, phase, and period the most likely regions for the two fits do not overlap. On this point we are in disagreement with ref. 21] which finds that the phase and period of the muon dataset can be in agreement with the DAMA signal. We believe that the source of this discrepancy is the choice of time origin which becomes important once the period is allowed to float.

3. A correlation analysis which is independent of any choice of functional form or fit to the datasets excludes the simple, yet very general model presented by ref. 21] for connecting the muon flux with the DAMA signal. It indicates that such a model would imply a much stronger degree of correlation than what is in fact observed in the data. Thereby, this analysis also puts to question the hypothesis 15, 17 itself that the variation of the muon flux has a casual connection with the one seen by DAMA.

Aside from these three difficulties, which depend only on the temporal structure of the two signals, there also seems to be some difficulty reconciling the amplitude of the muon modulations with that of the DAMA signal. On the face of it, the amplitude of the modulations observed by LVD seems to agree well with the $\sim 2 \%$ modulations as originally quoted by the DAMA collaboration. However, the full single-hit energy spectrum as measured in DAMA-LIBRA 9, seems to suffer from ${ }^{40} \mathrm{~K}$ contamination in the signal region. Moreover, it was recently pointed out in ref. 40] that the radioactive background rates in the DAMA experiment are likely larger than what was estimated by DAMA. If true, that would imply a smaller unmodulated amplitude of the putative signal and hence a larger modulation fraction. Thus, any model attempting to explain the DAMA signal as a result of the 
muon flux will require some sort of nonlinearity in the signal yield.

Given all of the above, we find the muon hypothesis in its current form rather unpersuasive. Any further investigations into this possibility should address the aforementioned difficulties.

With regards to the CoGeNT data our analysis concentrated on three different energy regions, $0.5-0.9 \mathrm{keV}_{\mathrm{ee}}$, $0.9-1.6 \mathrm{keV}_{\mathrm{ee}}$, and $1.6-3.0 \mathrm{keV}_{\mathrm{ee}}$. We find that the presence of annual modulations can only be firmly established in the $1.6-3.0 \mathrm{keV}_{\text {ee }}$ region. The other two regions require a somewhat more careful treatment of the data to remove the contribution from the cosmogenic activity which contributes to the power spectrum at long periods due to the long-lived isotopes involved. Nevertheless, none of the procedures we have followed for subtracting the cosmogenic contributions resulted in a statistically significant yearly modulation signal in the subtracted data. The phase analysis of the modulations shows them to peak around the middle of April, which is somewhat earlier than expected from DM recoils, but the uncertainty on this number is still rather large. Indeed, we find that the confidence intervals in the full two dimensional phase-period space are much too large for any significant statement to be made about the modulations observed.

As in the case of DAMA we find no significant correlation between the CoGeNT data set and the yearly modulation of the muon flux. In the absence of published measurements of the latter for the time-period of the CoGeNT run we used the atmospheric effective temperature inferred from climate data instead. The one-to-one relation between the variation in temperature and muon-flux allows us again to indirectly disfavor the muon hypothesis as it applies to the modulations seen by CoGeNT.

One can also wonder if muon induced backgrounds instead have a delayed signal, would that allow them to explain these dark matter anomalies? As shown in Appendix C production of long-lived radioactive isotopes does not result in any improvement. The largest allowed temporal shift of the signal peak is to the beginning of October. To complicate matters, the modulation amplitude of such a signal is always smaller than the muon amplitude. These limitations make it difficult to explain DAMA or CoGeNT with such muon backgrounds.

Finally, we discussed a new diagnostic for dark matter direct detection experiments. Since the expected recoil rate of dark matter against matter in the lab is a general periodic function with a fundamental period of one year, it also contains biannual modulations as well as higher harmonics. The relative amplitude of the velocity modulation is roughly $V_{\oplus} / 2 v_{\odot} \approx 0.06$, which is rather small. The higher harmonics in the recoil rate have similar phase to the annual modulation, but are generally suppressed. However, we presented a few cases where they might be observed given sufficient exposure. At the very least, the biannual mode can be looked for and limits on its power can be obtained in a straightforward fashion.

\section{ACKNOWLEDGMENTS}

We acknowledge helpful discussions with L. Dai, M. Kamionkowski, D. Morrissey and M. Pospelov. We thank J. Collar for assistance in interpreting the CoGeNT data.

\section{Appendix A: Bayesian Version of the Lomb-Scargle Periodogram}

In this appendix we produce a derivation of the LombScargle periodogram based on Bayesian analysis. This result was first derived in Ref. [41. We present it here in order to introduce the terminology, methodology, and notation used in deriving some of the other results, as well as in order for this paper to be as self-contained as possible.

We begin by considering the oscillatory model function $f$ with a single harmonic frequency $\omega$,

$$
f(t)=A \cos (\omega t)+B \sin (\omega t),
$$

as the hypothesis for the signal. The data points are therefore given by,

$$
d_{i}=f\left(t_{i}\right)+n_{i}
$$

where the data is taken to have zero mean $\langle d\rangle=0$. The noise is denoted by $n_{i}$ which is assumed to be normally distributed with zero mean and variance $\sigma^{2}$. Under the hypothesis A-1 the probability $P(d \|\{A, B, \omega\})$ of observing the data $d$ given the parameters $\{A, B, \omega\}$ is then directly proportional to the likelihood function

$$
\begin{aligned}
L(\{A, B, \omega\}, d) & =\prod_{i=1}^{N} \frac{1}{\sqrt{2 \pi \sigma^{2}}} \exp \left\{-\frac{\left[d_{i}-f\left(t_{i}\right)\right]^{2}}{2 \sigma^{2}}\right\} \\
& =\left(\frac{1}{\sqrt{2 \pi \sigma^{2}}}\right)^{N} \exp \left(-\frac{N Q}{2 \sigma^{2}}\right),
\end{aligned}
$$

where $N$ is the number of data points and

$$
\begin{aligned}
Q & =\left\langle d^{2}\right\rangle-\frac{2}{N}\left[A \sum_{i} d_{i} \cos \left(\omega t_{i}\right)+B \sum_{i} d_{i} \sin \left(\omega t_{i}\right)\right] \\
& +\frac{1}{N}\left[A^{2} \sum_{i} \cos ^{2}\left(\omega t_{i}\right)+B^{2} \sum_{i} \sin ^{2}\left(\omega t_{i}\right)\right. \\
& \left.+2 A B \sum_{i} \cos \left(\omega t_{i}\right) \sin \left(\omega t_{i}\right)\right]
\end{aligned}
$$

Bayes' theorem then allows us to obtain the posterior distribution $P(\{\omega, A, B\} \| d)$, i.e. the probability of the parameters $\{\omega, A, B\}$ given the data $d$,

$$
P(\{\omega, A, B\} \| d) \propto P(\{\omega, A, b\}) L(\{A, B, \omega\}, d) .(\mathrm{A}-5)
$$

Assuming a flat prior on the nuisance parameters $\mathrm{A}$ and B, we can integrate over A and B to obtain the posterior distribution of $\omega$ alone. This is a gaussian integral, 
and would be trivial except for the cross term between A and $\mathrm{B}$ in $Q$. This term can be eliminated with the introduction of a phase-shift, $\tau$, by defining $\tilde{t}_{i} \equiv t_{i}-\tau$ and using the fact that $\langle d\rangle=0$,

$$
\begin{aligned}
Q & =\left\langle d^{2}\right\rangle-\frac{2}{N}\left[A \sum_{i} d_{i} \cos \left(\omega \tilde{t}_{i}\right)+B \sum_{i} d_{i} \sin \left(\omega \tilde{t}_{i}\right)\right] \\
& +\frac{1}{N}\left[A^{2} \sum_{i} \cos ^{2}\left(\omega \tilde{t}_{i}\right)+B^{2} \sum_{i} \sin ^{2}\left(\omega \tilde{t}_{i}\right)\right] . \quad(\mathrm{A}-6)
\end{aligned}
$$

Effecting the gaussian integral over A and B gives,

$$
\begin{aligned}
P\left(\{\omega\} \| d_{i}\right) & \propto \int d A d B P(A, B) L(\{A, B, \omega\}, d) \\
& =\left(\frac{1}{\sqrt{2 \pi \sigma^{2}}}\right)^{N-2} \frac{1}{\sqrt{\sum_{i} \cos ^{2}\left(\omega \tilde{t}_{i}\right) \sum_{i} \sin ^{2}\left(\omega \tilde{t}_{i}\right)}} \\
& \times \exp \left\{-\frac{1}{2 \sigma^{2}}\left[N\left\langle d^{2}\right\rangle-\operatorname{LS}(\omega)\right]\right\},
\end{aligned}
$$

where the LS function is,

$$
\begin{aligned}
\operatorname{LS}(\omega) & =\frac{1}{\sum_{i} \cos ^{2}\left(\omega \tilde{t}_{i}\right)}\left[\sum_{i} d_{i} \cos \left(\omega \tilde{t}_{i}\right)\right]^{2} \\
& +\frac{1}{\sum_{i} \sin ^{2}\left(\omega \tilde{t}_{i}\right)}\left[\sum_{i} d_{i} \sin \left(\omega \tilde{t}_{i}\right)\right]^{2}
\end{aligned}
$$

The amplitude of the exponential in the posterior distribution, Eq. A-7], is a rather mild function of the frequency and so the most likely value for the frequency is the one which maximizes the LS function. This result is the Bayesian version of the usual frequentist LS statistics.

This approach lends itself to several generalizations and extensions. Ref. [41] derives the posterior for the frequency associated with a sinusoidal signal with general coefficients (not necessarily constants). It is also possible to derive the posterior on the phase of a sinusoidal signal following similar steps to the above.

\section{Appendix B: Cosmic muon flux}

In this section we discuss the connection between cosmic ray induced muon flux and its seasonal variation. Both, DAMA and CoGeNT, are operated deeply underground ( $>2000$ mwe) so that these detectors are well shielded from cosmic rays. Only muons with initial energies $\mathcal{O}(\mathrm{TeV})$ are able to reach the respective LNGS and Soudan Mine underground facilities. The highly energetic muons are sourced from primary meson ( $\pi$ 's, $K$ 's,...) decays which themselves are produced when cosmic rays hit the atmosphere. Since the chance for primary mesons to undergo further interactions grows with air density, there is an increasing competition between further degradation and decay when going to lower atmospheric levels.

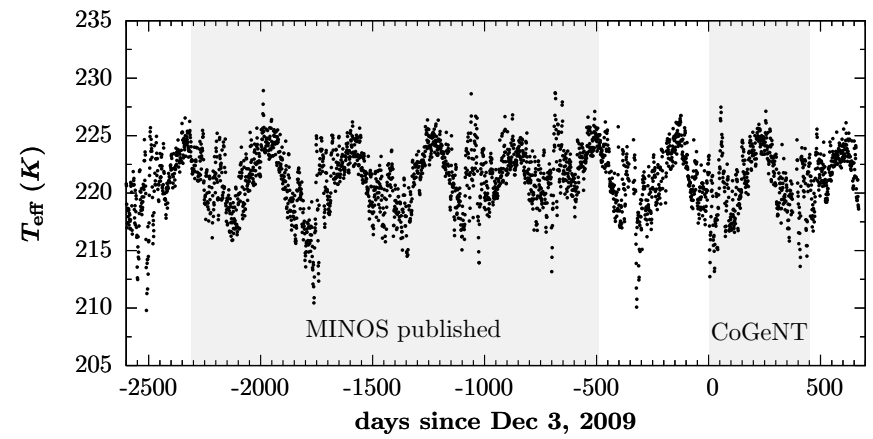

FIG. 12. Evolution of effective temperature parameter $T_{\text {eff }}$ at the Soudan site as a function of time. The gray shaded regions indicate the overlaps with data-taking periods of MINOS and CoGeNT as labeled.

The above chain of arguments leads to the following picture for the high-energy part of the muon flux: the production proceeds predominantly at stratospheric levels which are mainly subject to seasonal temperature (and thus air density) variations. The troposphere-with its daily modulation in temperature - only plays a minor role in determining the muon flux. The phenomenological relationship between muon flux and temperature can be written as

$$
\frac{\Delta I_{\mu}}{\left\langle I_{\mu}\right\rangle}=\alpha_{T} \frac{\Delta T_{\mathrm{eff}}}{\left\langle T_{\mathrm{eff}}\right\rangle},
$$

where $\left\langle I_{\mu}\right\rangle$ is the (average) integral underground flux (with variation $\Delta I_{\mu}$ ) and $\alpha_{T}$ is an effective temperature coefficient with inferred values $\alpha_{T} \simeq 0.93$ [20] and $\alpha_{T} \simeq 0.87$ [29] for the LNGS and Soudan Mine sites, respectively. The effective temperature $T_{\text {eff }}$ is obtained from the weighted average

$$
T_{\text {eff }}=\frac{\int_{0}^{\infty} d X T(X) W(X)}{\int_{0}^{\infty} d X W(X)}
$$

where $X$ is the atmospheric depth. The weight function $W(X)$ depends on parameters such as the amount of inclusive meson production in the forward fragmentation region, atmospheric attenuation parameters of mesons, and the muon spectral index. When computing $T_{\text {eff }}$ we use the expression for $W$ given in [29] which includes the contribution of pions as well as kaons.

The tight correlation between $\Delta I_{\mu}$ and $\Delta T_{\text {eff }}$ is firmly established by a whole range of independent measurements and at various geographic underground locations, such as at LNGS 18 20, at the Soudan Mine 29, or at the South Pole 31. For DAMA, the relevant LVD measurements are discussed in the introduction. Turning to the CoGeNT site, the MINOS far detector has recently reported measurements on the underground muon flux spanning a total of five years [29]. Unfortunately, the published data does not overlap with the CoGeNT run starting Dec 4, 2009 and lasting 458 days. The results are nevertheless relevant as they corroborate the 
tight relation between effective temperature and measured muon flux. In order to make statements about the muon background hypothesis for CoGeNT we have obtained climate data from the Integrated Global Radiosonde Archive (IGRA) for the nearby International Falls (MN) weather station [42. We compute daily $T_{\text {eff }}$ values by evaluating a discretized version of $(\overline{\mathrm{B}}-2)$ including only high-quality data reaching atmospheric pressure levels below $40 \mathrm{hPa}$. The result of this procedure is shown in Fig. 12 which presents effective temperature values for a time span covering both, MINOS and CoGeNT, as indicated. We note in passing that the same IGRA data set was also used by the MINOS collaboration itself to confirm $T_{\text {eff }}$ values obtained from another climate archive with good agreement.

\section{Appendix C: Activation of long-lived isotopes}

A loop-hole that exists in the analysis of assessing the viability of the muon hypothesis for DAMA and CoGeNT is the following: What if one significantly breaks the temporal coincidence between muon arrival time in and around the detector and the time at which the signal is produced. "Significantly" here means a shift in time which at least on the order of weeks and more. It is clear then that one cannot compare the time series of muon flux data with the one from DAMA and CoGeNT at face value.

The muon flux itself can give rise to cosmogenically activated isotopes. For example, the most inner shielding of the DAMA detector is made from massive copper so that for example a reaction ${ }^{65} \mathrm{Cu}+\mu^{+} \rightarrow{ }^{65} \mathrm{Zn}+\bar{\nu}_{\mu}$ with a delayed decay of ${ }^{65} \mathrm{Zn}$ with a half-life of 244 days is conceivable. Though the weak-scale cross section draws doubt on the efficiency of such a process, neutron capture reactions have cross sections many orders of magnitude larger. Indeed, it was one of the central points in Ref. [15] to point to the importance of $(n, \gamma)$ reactions for background considerations in direct detection experiments. Let us at this point not worry about the particular process but rather tackle the problem from the time series point of view. The question we are after is whether it is possible to induce a time-delay $\hat{t}$ in the signal such that it features a maximum in the year which is compatible with DAMA (and possibly by CoGenT.) As we have seen, the muon flux peaks at least a month later than the signals under consideration. Therefore, one requires $\hat{t} \gtrsim 300$ days.

For simplicity, let us model the muon flux as a sinosoidal function, $I_{\mu}(t)=I_{0}\left[1+I_{m} \cos (\omega t)\right]$ where we are choosing the origin of time to coincide with its maximum. Call $n$ the number density of a stable element and $n^{*}$ its "excited" state formed upon a generalized interaction with the muon flux and which decays with a lifetime of $\tau=\lambda^{-1}$. The abundance of $n^{*}$ is obtained by solving

$$
\frac{d n^{*}(t)}{d t}=-\lambda n^{*}(t)+S_{0}\left[1+S_{m} \cos (\omega t)\right]
$$

with an overall source term $S_{0}$ with modulating fraction $S_{m}$ for activation. In the physical picture, $S_{0}=\lambda_{\text {act }} n$, where $\lambda_{\text {act }}$ is the rate for activation and given by the product of the activating (neutron) flux times the cross section (with a potential average over the flux energy spectrum.) Clearly, $S_{0} \propto I_{0}$ holds with the same phase and modulation amplitude in Eq. (C-1) as in $I_{\mu}(t)$.

If the events seen by DAMA (or CoGeNT) are somehow related to the decay of the radioactive isotope then the event rate in the detector will be proportional to $R \propto \lambda n^{*}(t)$ which is just the usual relation for the number of radioactive decays. The rate equation Eq. (C-1) can be solved exactly,

$$
n^{*}(t)=C_{0} e^{-\lambda t}+S_{0} \tau+\tilde{S}_{m} \cos (\omega t-\theta),
$$

where $\tan \theta=\omega / \lambda$ and $\tilde{S}_{m}=S_{0} S_{m} / \sqrt{\lambda^{2}+\omega^{2}}$. The first term is transitory and vanishes for $t \gg \tau$. Therefore, the event rate will approach

$$
R \sim S_{0}+\lambda \tilde{S}_{m} \cos [\omega(t-\hat{t})],
$$

with the time delay, $\hat{t}=\theta / \omega$. The latter is always a positive quantity with a minimum of zero days and maximum of 91.25 days (quarter year). The phase delay is maximal $(\theta=\pi / 2)$ when the lifetime of the decay is much longer than one year $\tau \gg 1 \mathrm{yr} / 2 \pi$. Also, interestingly, the modulation amplitude is always smaller than the muon flux, since $\lambda \tilde{S}_{m} / S_{0}=\left(\lambda / \sqrt{\lambda^{2}+\omega^{2}}\right) I_{m}$. Thus, we see that this muon related background is not optimal for explaining DAMA or CoGeNT, since the maximum of the signal can only be shifted into the fall, but not further into spring as would be required by DAMA and/or CoGeNT, and the modulation amplitude of the signal is always reduced from the muon's amplitude.
[1] M. W. Goodman and E. Witten, Phys.Rev. D31, 3059 (1985).

[2] D. Tucker-Smith and N. Weiner, Phys.Rev. D64, 043502 (2001), arXiv:hep-ph/0101138 [hep-ph].

[3] D. S. Alves, S. R. Behbahani, P. Schuster, and J. G. Wacker, Phys.Lett. B692, 323 (2010), arXiv:0903.3945 [hep-ph].

[4] S. Chang, A. Pierce, and N. Weiner, JCAP 1001, 006
(2010), arXiv:0908.3192 [hep-ph].

[5] S. Chang, N. Weiner, and I. Yavin, Phys.Rev. D82, 125011 (2010), arXiv:1007.4200 [hep-ph].

[6] B. Feldstein, A. Fitzpatrick, and E. Katz, JCAP 1001, 020 (2010), arXiv:0908.2991 [hep-ph].

[7] A. Drukier, K. Freese, and D. Spergel, Phys.Rev. D33, 3495 (1986).

[8] K. Freese, J. Frieman, and A. Gould, Phys. Rev. D 37, 
3388 (1988)

[9] R. Bernabei et al., Eur. Phys. J. C56, 333 (2008) 0804.2741

[10] R. Bernabei et al., (2010), arXiv:1002.1028 [astroph.GA]

[11] C. Aalseth, P. Barbeau, J. Colaresi, J. Collar, J. Diaz Leon, et al., Phys.Rev.Lett. 107, 141301 (2011) arXiv:1106.0650 [astro-ph.CO].

[12] Z. Ahmed et al., (2009), 0912.3592

[13] Z. Ahmed et al., (2010) 1011.2482.

[14] E. Aprile et al., (2011), 1104.2549.

[15] J. P. Ralston, (2010), 1006.5255.

[16] ICARUS, internal report TM/03-01 (2003).

[17] D. Nygren, (2011), 1102.0815.

[18] M. Ambrosio et al. (MACRO Collaboration), Astropart.Phys. 7, 109 (1997).

[19] M. Selvi (LVD Collaboration), Proceedings of the 31st ICRC 1 (2009).

[20] D. D'Angelo (Borexino Collaboration), (2011), arXiv:1109.3901 [hep-ex].

[21] K. Blum, (2011), arXiv:1110.0857 [astro-ph.HE]

[22] P. J. Fox, J. Kopp, M. Lisanti, and N. Weiner, (2011), arXiv:1107.0717 [hep-ph]

[23] M. Farina, D. Pappadopulo, A. Strumia, and T. Volansky, JCAP 1111, 010 (2011), arXiv:1107.0715 [hep-ph],

[24] A. Schuster, Terrestrial Magnetism 3, 13 (1898).

[25] J. D. Scargle, ApJ 263, 835 (1982).

[26] J. Collar, Private communication.

[27] K. Nakamura et al. (Particle Data Group), J.Phys.G G37, 075021 (2010).

[28] C. Arina, J. Hamann, R. Trotta, and Y. Y. Wong, (2011), arXiv:1111.3238 [hep-ph].
[29] P. Adamson et al., Phys. Rev. D81, 012001 (2010), 0909.4012 .

[30] F. James and M. Roos, Comput.Phys.Commun. 10, 343 (1975)

[31] S. Tilav et al. (for the IceCube Collaboration), (2010), arXiv:1001.0776 [astro-ph.HE],

[32] C. Savage, K. Freese, P. Gondolo, and D. Spolyar, JCAP 0909, 036 (2009), arXiv:0901.2713 [astro-ph].

[33] J. D. Lewin and P. F. Smith, Astropart. Phys. 6, 87 (1996)

[34] M. Lisanti, L. E. Strigari, J. G. Wacker, and R. H. Wechsler, Phys.Rev. D83, 023519 (2011), arXiv:1010.4300 [astro-ph.CO]

[35] J. Bovy, D. W. Hogg, and H.-W. Rix, Astrophys.J. 704, 1704 (2009), arXiv:0907.5423 [astro-ph.GA]

[36] P. J. McMillan and J. J. Binney, (2009), arXiv:0907.4685 [astro-ph.GA]

[37] M. Reid, K. Menten, X. Zheng, A. Brunthaler, L. Moscadelli, et al., Astrophys.J. 700, 137 (2009) arXiv:0902.3913 [astro-ph.GA]

[38] M. C. Smith, G. Ruchti, A. Helmi, R. Wyse, J. Fulbright, et al., Mon.Not.Roy.Astron.Soc. 379, 755 (2007) arXiv:astro-ph/0611671 [astro-ph]

[39] E. J. Groth, ApJS 29, 285 (1975)

[40] V. Kudryavtsev, M. Robinson, and N. Spooner, Astropart.Phys. 33, 91 (2010).

[41] G. L. Bretthorst, "Frequency estimation and generalized Lomb-Scargle periodograms," in Statistical Challenges in Astronomy, edited by Feigelson, E. D. \& Babu, G. J. (2003) pp. 309-329.

[42] I. Durre, R. Vose, and D. Wuertz, Journal of Climate 19, 53 (2006). 